\title{
UM ESTUDO EXPLORATÓRIO DA TENDÊNCIA "ANTIOBESIDADE" ENTRE PROFESSORES E ESTUDANTES DE EDUCAÇÃO FÍSICA ITALIANOS
}

\author{
AN EXPLORATORY STUDY OF THE "ANTI-OBESITY" TREND AMONG ITALIAN \\ PHYSICAL EDUCATION TEACHERS AND STUDENTS
}

\begin{abstract}
UN ESTUDIO EXPLORATORIO DE LA TENDENCIA "ANTIOBESIDAD" ENTRE LOS PROFESORES Y ESTUDIANTES DE EDUCACIÓN FÍSICA ITALIANOS
\end{abstract}

Erica Gobbi*, Márcia Greguol**, Bruna Barboza Seron ${ }^{\star \star *}$, Attilio Carraro*

Palavras chave:

Obesidade.

Preconceito.

Protocolos.

Imagem corporal.

\begin{abstract}
Resumo: 0 objetivo desta pesquisa foi analisar as atitudes implícitas e explícitas sobre a obesidade em professores e estudantes de Educação Física. Dezoito professores e 45 estudantes preencheram quatro questionários: o Teste de Associação Implícita (IAT), que avalia por meio de categorizações os estereótipos e as associações cognitivas espontâneas; o questionário de Crandall para a avaliação do preconceito explícito sobre a obesidade; a Escala sobre a Orientação à Dominância Social (SDO); e o Questionário de Autodescrição Física - versão curta (PSDQ-s). Ambos os grupos reportaram preconceito significativo $(p<0,05)$, evidenciado pelos valores médios registrados pelo IAT. Os professores relataram valores significativamente maiores no preconceito implícito e valores mais baixos na SDO comparados aos estudantes. A Orientação à Dominância Social mostrou-se uma variável chave na presença do preconceito. Este estudo pode ser um ponto de partida para futuras pesquisas focadas na redução do preconceito por meio de protocolos de intervenção específicos.
\end{abstract}

Keywords:

Obesity.

Prejudice.

Protocols.

Body image.

Palabras clave:

Obesidad.

Prejuicio.

Protocolos

Imagen corporal. estudiantes completaron cuatro cuestionarios: el Test de Asociación Implícita (IAT), que
Abstract: This study looked into Physical Education teachers' and students' implicit and explicit attitudes toward obesity. Eighteen teachers and 45 students completed four questionnaires: the Implicit Association Test (IAT), which assesses stereotypes and spontaneous cognitive associations through categorizations; The Crandall questionnaire for the evaluation of explicit prejudice about obesity; The Social Dominance Orientation Scale (SDO); and the Physical Self-Description Questionnaire - short version (PSDQ-s). Both groups reported significant prejudice $(p<0.05)$, evidenced by the mean values recorded on the IAT. Teachers reported significantly higher values than students on implicit prejudice and lower values on SDO. Social Dominance Orientation proved to be a key variable in the presence of prejudice. This study may be a starting point for future research focused on reducing prejudice through specific intervention protocols.

Resumen: El objetivo de este estudio fue analizar las actitudes implícitas y explícitas sobre la obesidad entre profesores y estudiantes de educación física. Dieciocho profesores y 45 evalúa estereotipos y asociaciones cognitivas espontáneas; el cuestionario de Crandall para evaluar el prejuicio explícito acerca de la obesidad; la Escala de Orientación a la Dominancia Social (SDO); y el Cuestionario de Autoconcepto Físico - versión corta (PSDQ-s). Ambos grupos reportaron perjuicio significativo $(p<0,05)$, como se evidencia por los valores medios registrados por el IAT. Los profesores reportaron valores significativamente más altos en el prejuicio implícito y valores más bajos en la SDO en comparación con los estudiantes. La Orientación a la Dominancia Social demostró ser una variable clave en la presencia de perjuicios. Este estudio puede ser un punto de partida para futuras investigaciones enfocadas en la reducción del perjuicio a través de protocolos de intervención específicos.
*Universidade de Pádova, Itália. Pádova, Itália.

E-mail: erica.gobbi@unipd.it; attilio.carraro@unipd.it

**Universidade Estadual de Londrina. Londrina, PR, Brasil.

E-mail : mgreguol@gmail.com

${ }^{* \star *}$ Universidade Federal de Santa Catarina. Florianópolis, SC, Brasil. E-mail : bruna.seron@ufsc.br

Recebido em: 08-05-2017 Aprovado em: 19-06-2017

(c) (i) (2) Licence 


\section{INTRODUÇÃO}

A obesidade é uma condição determinada por excesso de massa gorda distribuída de maneiras diferentes nas várias regiões corporais, podendo causar problemas de saúde como diabetes melito, doenças coronarianas e certos tipos de câncer (WHO, 2016). Há algumas décadas, seja nos países desenvolvidos, seja naqueles em desenvolvimento, registra-se um aumento progressivo da prevalência de obesidade, tanto nos adultos como nas crianças: estima-se que em 2014 mais de 1,9 bilhão de adultos e 41 milhões de crianças reportavam uma condição de sobrepeso ou obesidade (WHO, 2016). Além da quantidade substancial de despesas dos serviços de saúde associadas à obesidade (CAWLEY; MEYERHOEFER, 2012), mais escondidos e menos questionados mostram-se ser os custos sociais e psicológicos que essa condição determina.

Muitas pessoas obesas relatam ter sofrido tratamento negativo por parte de vários profissionais, como, por exemplo: nutricionistas (OBERRIEDER et al., 1995), estudantes de medicina (WIGTON; MCGAGHIE, 2001), médicos (HEBL; XU, 2001), enfermeiros (MARONEY; GOLUB, 1992) e outros especialistas em obesidade (SCHWARTZ et al., 2003; TEACHMAN; BROWNELL, 2001). A discriminação das pessoas obesas já é difundida em vários contextos de vida, inclusive no ambiente de trabalho (ROTHBLUM; MILLER; GARBUTT, 1988), no qual existe uma disparidade nas perspectivas de carreira e na obtenção de promoções (PAGAN; DAVILA, 1997), e no contexto escolar, no qual emergem comportamentos de rejeição e marginalização (CRANDALL, 1991; NEUMARK-SZTAINER; STORY; FAIBISCH, 1998). A estigmatização da obesidade determina consequências por vezes muito profundas em nível psicoemotivo, por exemplo: sobre crianças de nove anos clinicamente em sobrepeso revelase uma significativa redução da autoestima, a convicção de ser motivo de chacota, de ter menos amigos e de ser excluído dos jogos e dos esportes essencialmente por causa de seu peso (PIERCE; WARDLE, 1997).

No ambiente escolar, os estudantes com sobrepeso ou obesos encontram maiores obstáculos quando comparados aos colegas eutróficos para a prática de atividade física: podem manifestar uma vergonha evidente, uma preocupação por sua saúde, uma exagerada atenção acerca da perda de peso, ser objeto de vitimização (PUHL; LUEDICKE, 2012; SCARPA et al., 2012), além de relacionar-se negativamente com situações de exercício físico, tendo em vista as experiências precedentes (BALL; CRAWFORD; OWEN, 2000; LYONS, MILLER, 1999). Alguns dados mostram como as crianças com obesidade, comparadas aos colegas eutróficos, reportam mais dias de ausência na escola (GEIER et al., 2007), o que se torna para essas crianças um obstáculo no processo de aprendizagem. Outra barreira constante à prática de atividade física por crianças e jovens em situação de obesidade é a discriminação e o preconceito por parte da figura dos educadores (FAITH et al., 2002; LYONS; MILLER, 1999).

Segundo alguns especialistas, a escola é um local-chave para a condução de intervenções focadas na prevenção da obesidade durante o período de desenvolvimento (HOELSCHER et al., 2013; USDHHS, 2010). No entanto, o que aconteceria se os professores fossem os primeiros a demonstrar uma atitude negativa a respeito de crianças e adolescentes em condição de obesidade? Entre esses profissionais, os professores de Educação Física (EF) desempenham um papel relevante na promoção da atividade física 
durante o desenvolvimento e é importante que consigam envolver todos os estudantes, mantendo o olhar inclusivo e equitativo. Esse grupo de professores é de particular interesse, uma vez que foi constatado que apresentam risco aumentado para a manifestação de burnout (CARRARO; GOBBI; MOĖ, 2016; CARRARO et al., 2010), uma condição que compromete a eficácia didática e que poderia desta forma acentuar as atitudes negativas no que se refere aos estudantes com obesidade.

A partir dessas premissas, o objetivo deste estudo foi explorar e confrontar as atitudes implícitas e explícitas no que diz respeito à obesidade por parte de professores de Educação Física no ensino fundamental e estudantes de um curso de Educação Física, os quais poderiam escolher em também tornarem-se professores de Educação Física Escolar. Foram questionadas ainda as relações entre o preconceito e duas variáveis psicossociais: a orientação à dominância social e a descrição do autoconceito físico. A hipótese inicial era de que entre os entrevistados houvesse uma atitude negativa no que diz respeito às pessoas obesas e que, confirmando os posicionamentos da literatura, fossem observadas relações positivas entre o preconceito e a orientação à dominância social e a descrição do autoconceito físico.

\section{MÉTODO}

\subsection{Participantes}

A pesquisa foi conduzida envolvendo dezoito professores de Educação Física do ensino fundamental da região do Vêneto, Itália (sete homens e 11 mulheres, com idade média de 51.7 \pm 5.9 anos), e 45 estudantes do curso de licenciatura em Educação Física da Universidade de Pádova, Itália (17 homens e 28 mulheres, com idade média de $24.6 \pm 1.9$ anos). Os participantes aderiram à participação no estudo assinando um termo de consentimento livre e esclarecido, e a realização do estudo foi aprovada pelo Comitê de Ética em Pesquisa local.

\subsection{Instrumentos}

A bateria de questionários inclui duas tarefas cronometradas do Teste de Associação Implícita (IAT) para a avaliação do preconceito implícito, o questionário Crandall para a avaliação do preconceito explícito sobre a obesidade, um questionário sobre a orientação para a dominância social (SDO) e uma descrição do autoconceito físico para avaliar dois constructos psicossociais correlatos à presença do preconceito.

\subsubsection{Teste de Associação Implícita}

O Teste de Associação Implícita, IAT (GREENWALD; MCGHEE; SCHWARTZ, 1998), é um instrumento para a medida de associações implícitas entre conceitos-alvo (ex: homem/mulher) ou entre conceitos e atributos de avaliação (ex: bom/mau, preguiçoso/ motivado). Trata-se de uma avaliação a ser realizada em um tempo predeterminado que inicialmente era usada para revelar o preconceito no que diz respeito a diferentes grupos sociais (GREENWALD; MCGHEE; SCHWARTZ, 1998) e que foi sucessivamente utilizada 
para avaliar o preconceito implícito no que se refere à obesidade em diferentes populações, inclusive estudantes e profissionais de saúde (SCHWARTZ et al., 2003; TEACHMAN; BROWNELL, 2001).

A versatilidade do IAT foi demonstrada também por meio do desenvolvimento de uma forma impressa do instrumento (LOWERY; HARDIN; SINCLAIR, 2001; TEACHMAN et al., 2003), a qual mostrou-se de mais fácil aplicação na pesquisa desenvolvida e que já foi utilizada em estudos precedentes para avaliar o preconceito implícito no que diz respeito à obesidade em populações de profissionais que atuam em serviços de saúde (CHAMBLISS; FINLEY; BLAIR, 2004; TEACHMAN; BROWNELL, 2001).

Foram apresentadas aos participantes duas tarefas do IAT para revelar a presença de associações implícitas dos atributos de avaliação motivado/preguiçosoe esperto/burrocom as categorias-alvo pessoas obesas e pessoas magras (Tabela 1). Os atributos foram coletados e traduzidos de estudos precedentes que demonstraram o que tipicamente representa os estereótipos comuns evidenciados sobre as pessoas em situação de sobrepeso e obesidade (SCHWARTZ et al., 2003; TEACHMAN, et al., 2003; TEACHMAN; BROWNELL, 2001).

Tabela 1 - Categorias alvo e categorias atributivas para o Teste de Associação Implícita.

\begin{tabular}{lccc}
\hline & & & \\
& & & \\
CATEGómulos a serem classificados \\
Pessoas obesas & Obeso & Gordo & Largo \\
Pessoas magras & Magro & Esquelético & Fino \\
\hline CATEGORIAS ATRIBUTIVAS (Primeira tarefa) & & & \\
Preguiçoso & Lento & Preguiçoso & Indolente \\
Motivado & Determinado & Motivado & Entusiasta \\
\hline CATEGORIAS ATRIBUTIVAS (Segunda tarefa) & & & \\
Esperto & Inteligente & Esperto & Brilhante \\
Burro & Ignorante & Burro & Enfadonho \\
\hline
\end{tabular}

Fonte: dados da pesquisa

A tarefa dos participantes consistia em categorizar os itens apresentados o mais rapidamente possível, classificando-os nas categorias supraordenadas (alvo e atributiva): foram apresentadas duas tarefas de categorização, para cada tarefa era apresentado também o acoplamento inverso das categorias e os participantes tinham à disposição 30 segundos de tempo para cada uma das tarefas. Cada teste foi formulado em uma página que foi estruturada com uma tabela de três colunas. Na coluna central eram apresentados os estímulos a serem classificados e as duas colunas laterais representavam as categorias supraordenadas dos estímulos de maneira acoplada (exemplo na Figura 1). Geralmente os entrevistados achavam mais fáceis as tarefas do IAT quando as categorias-alvo vinham apresentadas em associação a categorias atributivas que respondiam às suas associações cognitivas implícitas: no tempo predeterminado se consegue categorizar corretamente mais estímulos (cometem-se menos erros). 
Figura 1 - Exemplo de duas tarefas completas do IAT, que mede as associações implícitas entre o conceito de pessoa obesa e pessoa magra com as categorias de estímulos atributivos motivado/preguiçoso.

\begin{tabular}{|l|c|c|}
\hline $\begin{array}{c}\text { PESSOAS MAGRAS } \\
\text { MOTIVADO }\end{array}$ & $\begin{array}{c}\text { PESSOAS OBESAS } \\
\text { PREGUIÇOSO }\end{array}$ \\
\hline & Obeso & \\
\hline & Indolente & \\
\hline & Magro & \\
\hline & Entusiasta & \\
\hline & Largo & \\
\hline & Preguiçoso & \\
\hline & Gordo & \\
\hline & Motivado & \\
\hline & Fino & \\
\hline & Determinado & \\
\hline & Esquelético & \\
\hline
\end{tabular}

Fonte: Greenwald; Mcghee; Schwartz (1998)

\subsubsection{Medida "antiobesidade" explícita}

Para analisar o preconceito explícito foi utilizado o questionário proposto por Crandall (1994), para o qual foi feita tradução e adaptação transcultural. O questionário é constituído por 13 itens, subdivididos em três subescalas, para as quais o participante é solicitado a expressar seu grau de concordância de acordo com uma escala Likert, variando de 1 (discordo fortemente) a 9 (concordo fortemente). A subescala "antipatia" (itens 1 a 7 e pontuação de 7 a 63) avalia a aversão de uma pessoa no que diz respeito à obesidade; a subescala "medo da obesidade" (itens 8 a 10 e pontuação de 3 a 27) identifica o nível de preocupação de uma pessoa em ganhar peso; já a subescala "força de vontade" (itens 11 a 13 e pontuação de 3 a 27) revela quanta importância se dá à crença de que a obesidade seja um problema de falta de controle pessoal, isto é, que seja fruto dos próprios erros da pessoa. Já a pontuação total pode variar de 13 a 117. Em todas as situações, uma maior pontuação corresponde à presença de estigma antiobesidade.

\subsubsection{SDO}

A escala sobre a Orientação à Dominância Social, SDO, (DI STEFANO; ROCCATO, 2005) constitui-se em um instrumento de avaliação validado que se refere à ampla teoria da dominância social, desenvolvida na tentativa de fornecer uma explicação sobre a natureza dos conflitos entre as categorias sociais. É uma escala de 17 itens nos quais os participantes exprimem o grau de concordância mediante uma escala Likert partindo de 1 (em forte discordância) até 7 (em forte concordância). Além disso, a escala mostra-se racionalmente equilibrada pela presença de sete itens invertidos. A pontuação final varia de 17 a 119, sendo que um resultado mais alto corresponde a uma maior orientação à dominância social. 


\subsubsection{PSDQ-S}

Foi utilizada a versão curta do Questionário de Descrição do Autoconceito Físico, PSDQ-s, (PEART; MARSH; RICHARDS, 2006; versão italiana de SCARPA et al., 2010). 0 questionário validado compreende 43 itens (dez itens invertidos) e avalia nove componentes específicos e dois componentes globais do autoconceito físico. Os nove componentes específicos são Força, Obesidade, Atividade Física, Resistência, Habilidades Esportivas, Coordenação, Saúde, Aspecto e Flexibilidade; os dois componentes globais são o Autoconceito Físico Global e a Autoestima Global. Cada subescala é composta por quatro itens, exceto as escalas de Saúde e Obesidade, constituídas por cinco e três itens, respectivamente. Para cada afirmação, o participante era solicitado a utilizar uma escala de resposta do tipo verdadeiro/ falso, na qual 1 corresponde a falso e 6 a verdadeiro. As pontuações finais correspondem à soma das pontuações indicadas em cada item. É possível obter também a pontuação total do PSDq-s (que varia de 43 a 258 pontos), na qual um valor mais alto corresponde a uma melhor descrição do autoconceito físico.

\subsection{Procedimentos}

Os professores foram contatados e entrevistados individualmente na própria escola em que atuavam, levando cerca de 20 minutos para completar todos os instrumentos. Já para os estudantes os questionários foram aplicados durante 20 minutos de uma aula, em um horário pré-agendado.

Após a entrega do questionário ao participante, eram dadas as instruções de não abrir o envelope, uma vez que os primeiros testes eram tarefas com tempo predeterminado (cronometrado). Procedia-se com as instruções necessárias e com o preenchimento de uma tarefa do IAT para experimentação (foram escolhidas as categorias alvo para a experimentação: flores e insetos e aquelas atributivas: bom/mal), de forma que os participantes pudessem se familiarizar com as modalidades e os mecanismos de preenchimento necessários para a execução correta da tarefa. Procedia-se então com as tarefas cronometradas: 25 segundos para cada teste do IAT: um pesquisador dava a partida e interrompia o teste ao final do tempo, de forma que o participante categorizava o maior número possível de estímulos apresentados no limite de tempo concedido. Terminados os questionários cronometrados, os participantes eram solicitados a preencher de maneira autônoma os questionários sucessivos, prestando atenção para responder da maneira mais sincera e pessoal possivvel e para não omitir respostas.

\subsection{Análise Estatística}

Em primeiro lugar foi analisada a presença de preconceito implícito em ambos os grupos por meio de um teste t para uma amostra, verificando se a pontuação do IAT foi significativamente diferente do valor absoluto 0 (o qual indicava a ausência de preconceito). Em se verificando preconceito, foi utilizado um teste t pareado para comparar os resultados das tarefas do IAT de todo o grupo nas duas situações de categorização: quando a categoria atributiva era associada a estímulos negativos e positivos. Para avaliar a presença de diferenças entre professores e estudantes em todas as variáveis investigadas foi efetuado um teste $t$ para amostras independentes. Já para evidenciar as relações entre o preconceito implícito e 
explícito e as variáveis psicossociais foi utilizada a análise de correlação de Pearson. Os dados descritivos são apresentados como valores médios \pm desvio padrão e a significância adotada foi de $p<0.05$.

\section{RESULTADOS}

No que se refere ao preconceito implícito, foram calculados os valores médios das tarefas do IAT nas quais a categoria-alvo pessoa obesa estava em associação às categorias atributivas negativas preguiçoso e burro e àquelas positivas motivado e esperto. Ambos os grupos reportaram no teste t para amostra única uma significativa presença de preconceito implícito referido à associação pessoa obesa/preguiçosa $(t=3.19$ e $t=7.90 \mathrm{com} p<.01$ para os professores e estudantes, respectivamente). Analisando esse preconceito, todo o grupo de participantes conseguiu categorizar corretamente um número significativamente maior de estímulos quando a categoria-alvo pessoa obesa era associada a estímulos negativos (preguiçosa), comparado quando era associada a estímulos positivos (motivada) ( $t=8.18, p$ $<$.001). Os valores médios registrados em ambas as tarefas de categorização foram: obeso/ preguiçoso $10.27 \pm 1.25$ e obeso motivado $7.92 \pm 2.28$; $p<.001$; obeso/burro $9.62 \pm 1.81 \mathrm{e}$ obeso/esperto $9.79 \pm 1.94$.

O teste t para amostras independentes demonstrou diferenças significativas entre os professores e estudantes em algumas variáveis investigadas (estatística descritiva na Tabela 2). Em particular, os professores relatam um preconceito implícito maior comparado aos estudantes no que se refere ao estigma pessoa obesa/burra $(t=2.33 ; p<.05)$, e uma orientação à dominância social significativamente inferior $(t=-8.74 ; p<.001)$. Não foram vistas diferenças significativas nas variáveis preconceito explícito e PSDQ total, sendo que este último evidenciou pontuação elevada a respeito da descrição do autoconceito físico para os dois grupos.

Tabela 2 - Estatística descritiva para professores e estudantes para todas as variáveis consideradas.

\begin{tabular}{lcc}
\hline VARIÁVEL (possível pontuação) & $\begin{array}{c}\text { Professores } \\
(n=18) \\
\text { Média } \pm \mathrm{DP}\end{array}$ & $\begin{array}{c}\text { Estudantes } \\
(n=45) \\
\text { Média } \pm \mathrm{DP}\end{array}$ \\
\hline IAT & $2.1 \pm 2.7$ & \\
$\quad$ Obeso/preguiçoso & $0.9 \pm 1.9$ & $2.5 \pm 2.1$ \\
$\quad$ Obeso/burro & $43.3 \pm 13.7$ & $-0.6 \pm 2.4$ \\
SDO (17 a 119) & & $68.1 \pm 8.5$ \\
Preconceito explícito & $16.3 \pm 10.5$ & \\
$\quad$ Antipatia (7 a 63) & $11.0 \pm 5.5$ & $19.1 \pm 9.3$ \\
$\quad$ Medo da obesidade (3 a 27) & $17.3 \pm 5.6$ & $13.1 \pm 6.8$ \\
Força de vontade (3 a 27) & $44.7 \pm 13.9$ & $19.7 \pm 3.8$ \\
$\quad$ Total (13 a 117) & $211.9 \pm 32.1$ & $51.8 \pm 14.9$ \\
PSDQ total (43 a 258) & $0.96 .9 \pm 24.4$ \\
\hline
\end{tabular}

Nota. $\mathrm{DP}$ = Desvio Padrão; IAT = Teste de Associação Implícita; SDO = Orientação para dominância social; $P S D Q$ = descrição do autoconceito físico Fonte: dados da pesquisa

Considerando os participantes como grupo único de pessoas que se ocupam da Educação Física, foram analisadas as correlações entre as variáveis. Particularmente da 
correlação de Pearson emerge uma correlação significativa entre o preconceito implícito para a associação pessoa obesa/preguiçosa e a subescala aspecto do PSDQ-s ( $r=-0.27 ; p<.05)$. No que se refere à orientação à dominância social, houve relação tanto com o preconceito explícito quanto com o PSDQ-s (Tabela 3). Ainda foram vistas algumas correlações significativas de interesse entre as subescalas do PSDQ-s e as dimensões do preconceito explícito: em particular, a dimensão antipatia em relação ao obeso foi significativamente correlacionada à subescala atividade $(r=0.30 ; p<.05)$ e à subescala aspecto $(r=0.37 ; p<.05)$ do PSDQ-s; a dimensão medo do obeso correlacionou-se negativamente com as subescalas obesidade $(r$ $=-0.32 ; p<.05)$ e físico global $(r=-0.33 ; p<.01)$; a dimensão força de vontade de preconceito explícito relacionou-se de maneira significativa à subescala habilidade esportiva $(r=0.28 ; p<$ .05) do PSDQ-S.

Tabela 3 - Correlação de Pearson entre a orientação para a dominância social, o preconceito explícito e a descrição da autoimagem.

\begin{tabular}{ccccccc}
\hline & & Antipatia & Medo do obeso & $\begin{array}{c}\text { Força de } \\
\text { vontade }\end{array}$ & Crandall total & $\begin{array}{c}\text { PSDQ } \\
\text { total }\end{array}$ \\
\hline \multirow{2}{*}{ SDO } & Pearson's $r$ & .33 & .26 & .28 & .41 & .297 \\
& $\mathrm{P}$ & .009 & .037 & .026 & .001 & .018 \\
\hline
\end{tabular}

Nota. $\mathrm{SDO}=$ Orientação para a dominância social; $\mathrm{PSDQ}=$ Descrição da autoimagem.

\section{DISCUSSÃO}

O presente estudo se propôs a investigar a presença de preconceito implícito e explícito sobre a obesidade de um grupo de professores e estudantes de Educação Física em relação também à orientação à dominância social e à descrição do autoconceito físico. A partir da análise dos dados verificou-se, como se havia hipotetizado, que em ambos os grupos de participantes houve uma atitude negativa no que se refere às pessoas obesas. Estes dados são comparáveis em amplitude àqueles de estudantes de Educação Física e de profissionais de saúde pesquisados nos Estados Unidos (CHAMBLISS; FINLEY; BLAIR, 2004; TEACHMAN; BROWNELL, 2001). A partir da análise, emerge ainda que a associação implícita entre "pessoas obesas" e "preguiça" é mais forte do que aquela entre "pessoas obesas" e "burrice": para a categoria entrevistada as limitações de movimento que as pessoas em estado de sobrepeso ou obesidade encontram são provavelmente fundamentais para sua condição. Sobre este aspecto, reforça-se que os professores de Educação Física relatam um preconceito significativamente maior na associação implícita "pessoa obesa/burra" do que os estudantes; talvez a destreza e as habilidades físicas venham associadas com o fato de ser inteligente, em face de alguma experiência profissional vivenciada nas escolas.

Outra evidência a ser destacada entre os grupos dos professores e dos estudantes é o baixo nível dos primeiros na orientação à dominância social. Este ponto pode encontrar uma interpretação no fato de que os estudantes estejam ainda em formação, reconhecendo, portanto, mais expectativas profissionais futuras e, assim, apresentando um nível de orientação à dominância social mais elevado do que aquele percebido pelos professores, os quais em geral relatam um status social inferior àquele da maior parte dos outros profissionais (BIDDLE; GOOD; GOODSON, 2013). 
A partir das análises de correlação, foram observadas relações interessantes que em parte confirmam a literatura. Em destaque, salienta-se 0 fato de que as atitudes implícitas e explícitas não se correlacionam: o preconceito implícito avaliado por meio das tarefas do IAT não se correlacionou com o preconceito explícito registrado com o questionário de Crandall. Esse dado pode confirmar a necessidade de técnicas de investigação implícita sobre os comportamentos pessoais, uma vez que, ao se utilizar técnicas explícitas, duas questões problemáticas podem ocorrer: em primeiro lugar, a conveniência das respostas e a consequente distorção daquilo que as pessoas afirmam explicitamente; e, em segundo lugar, os limites na capacidade introspectiva do respondente, que muitas vezes não consegue fornecer um relato detalhado e verdadeiro dos conteúdos cognitivos de interesse (ZOGMAISTER; CASTELLI, 2006).

A orientação à dominância social foi significativamente correlacionada com todas as dimensões do preconceito explícito e com a descrição do autoconceito físico. Esse fato confirma o que foi reportado por O'Brien e Colleghi (2007) e requer uma reflexão sobre as razões pelas quais os indivíduos com elevada SDO tendem também a ser prevenidos na alocação de recursos (SIDANIUS; PRATTO, 1999) e, assim, a demonstrar comportamentos não inclusivos.

De pesquisas anteriores também já havia sido evidenciado que uma elevada autoestima no que se refere aos aspectos físicos era correlacionada fortemente a uma tendência "antiobesidade" (O'BRIEN; HUNTER; BANKS, 2007). Sobre este aspecto, os resultados do presente estudo confirmam as direções da literatura: as dimensões do preconceito explícito antipatia, medo do obeso e as atribuições de importância à força de vontade apresentaram relação significativa com algumas subescalas do PSDQ-s, que representam a percepção do próprio aspecto e da própria habilidade esportiva. Destaca-se que o medo do obeso e de ganhar peso apresentou uma relação negativa com a subescala relacionada à obesidade e à percepção do físico global.

Os indivíduos participantes do presente estudo se ocupam ou se ocuparão do ensino da Educação Física em diversos contextos (escolas, clubes esportivos, academias etc.). Os resultados sugerem que, não obstante seus percursos formativos e seu profissionalismo, o preconceito implícito e o explícito a respeito das pessoas obesas estão presentes e podem comprometer a eficácia e a qualidade das escolhas educativas e comportamentais.

Os resultados sugerem que são necessárias algumas estratégias para contrastar essas atitudes negativas sobre a obesidade, justamente por conta da importância que esses profissionais apresentam nos serviços voltados às pessoas obesas ou com sobrepeso. Particularmente, os cursos de formação em Educação Física poderiam aproximar os estudantes do problema, desenvolvendo a consciência sobre a existência de atitudes implícitas e sobre quais seriam as reais causas da obesidade e quais são os desconfortos psicossociais que a categoria estigmatizada vive. Além disso, poderiam ser previstos cursos de atualização para os professores em serviço, de modo a oferecer suporte às suas escolhas didáticas, por meio de uma nova perspectiva de inclusão e promoção da prática de atividade física acessível a todos (GREGUOL; GOBBI; CARRARO, 2013).

A pesquisa realizada apresenta a limitação imposta pelo número pequeno de participantes no grupo dos professores, no entanto é um estudo exploratório e os instrumentos utilizados se revelaram sensíveis ao objetivo: conseguiram individualizar a presença de atitudes negativas no que se refere à obesidade (provavelmente presentes em um nível 
inconsciente) por parte dos entrevistados. Assim, a pesquisa pode se revelar um ponto de partida para estudos futuros que procurem investigar sobre os determinantes do preconceito e, consequentemente, sobre a redução do preconceito implícito, por meio da experimentação de soluções e protocolos inovadores.

\section{REFERÊNCIAS}

BALL, Kylie; CRAWFORD, David; OWEN, Neville. Too fat to exercise? Obesity as a barrier to physical activity. Australian and New Zealand Journal of Public Health, v.24, n. 3, p.331-333, 2000.

BIDDLE, Bruce J.; GOOD, Thomas L.; GOODSON, Ivor (Eds.). International Handbook of Teachers and Teaching. Berlin: Springer Science \& Business Media, 2013. v.3.

CARRARO, Attilio; GOBBI, Erica; Moè, Angelica. More gyms or more psychological support? Preventing burnout and supporting job satisfaction in physical education teachers. Sport Sciences for Health, v.13, n.1, p.52-62, 2017.

CARRARO, Attilio et al. Burnout and self-perceptions of physical fitness in a sample of Italian physical education teachers. Perceptual and Motor Skills, v.111, n. 3, p.790-798, 2010.

CAWLEY, John; MEYERHOEFER, Chad. The medical care costs of obesity: an instrumental variables approach. Journal of Health Economics, n.31, n.1, p.219-230, 2012.

CHAMBLISS, Heather O.; FINLEY, Carrie E.; BLAIR, Steven N. Attitudes toward obese individuals among exercise science students. Medicine and Science in Sports and Exercise, v. 36, n. 3, p. 468-474, 2004.

CRANDALL, Christian S. Do heavy-weight students have more difficulty paying for college? Personality and Social Psychology Bulletin, v.17, n.6, p. 606-611,1991.

CRANDALL, Christian S. Prejudice against fat people: ideology and self-interest. Journal of Personality and Social Psychology, v.66, n.5, p.882-94, 1994.

DI STEFANO, Giovanni; ROCCATO, Michele. Una banca di item per misurare l'orientamento alla dominanza sociale in Italia. TPM, v.12, p.1-16, 2005.

FAITH, Myles S. et al. Weight criticism during physical activity, coping skills, and reported physical activity in children. Pediatrics, v.110, n.2, Pt. 1:e23, 2002.

GEIER, Andrew B. et al. The relationship between relative weight and school attendance among elementary schoolchildren. Obesity, v.15, n.8, p. 2157-2161, 2007.

GREENWALD, Anthony G.; MCGHEE, Debbie E.; SCHWARTZ, Jordan K. L. Measuring individual differences in implicit cognition: the Implicit Association Test. Journal of Personality and Social Psychology, v.74, n.6, p.1464-1480, 1998.

GREGUOL, Márcia; GOBBI, Erica; CARRARO, Attilio. Formação de professores para a educação especial: uma discussão sobre os modelos brasileiro e italiano. Revista Brasileira de Educação Especial, v.19, n.3, p.307-324, 2013. 
HEBL, Michelle R.; XU, Zhenghao. Weighing the care: physicians' reactions to the size of a patient. International Journal of Obesity and Related Metabolic Disorders, v.25, n.8, p.1246-1252, 2001.

HOELSCHER, Deanna M. et al. Position of the Academy of Nutrition and Dietetics: interventions for the prevention and treatment of pediatric overweight and obesity. Journal of the Academy of Nutrition and Dietetics, v.113, n.10, p. 1375-1394, 2013.

LATNER, Janet D.; STUNKARD, Albert J. Getting Worse: The stigmatization of obese children. Obesity Research, v.11, n.3, p.452-456, 2013.

LOWERY, Brian S.; HARDIN, Curtis D.; SINCLAIR, Stacey. Social influence effects on automatic racial prejudice. Journal of Personality and Social Psychology, v.81, n.5, p.842-855, 2001.

LYONS, Pat ; MILLER, Wayne C. Effective health promotion and clinical care for large people. Medicine and Science in Sports and Exercise, v.31, n.8, p.1141-1146, 1999.

MARONEY, Diane; GOLUB, Sharon. Nurses' attitudes toward obese persons and certain ethnic groups. Perceptual and Motor Skills, v.75, n.2, p.387-391, 1992.

MITCHELL, Jonathan A. et al. Sedentary behavior and obesity in a large cohort of children. Obesity, v.17, n.8, p.1596-1602, 2009.

NEUMARK-SZTAINER, Dianne; STORY, Mary; FAIBISH, Loren. Perceived stigmatization among overweight African-American and Caucasian adolescent girls. Journal of Adolescent Health, v.23, n.5, p.264-270, 1998.

O'BRIEN, Kerry S.; HUNTER, John A.; BANKS, Mike. Implicit anti-fat bias in physical educators: physical attributes, ideology and socialization. International Journal of Obesity,v.31, n.2, p.308-314, 2007.

OBERRIEDER, Heidi et al. Attitude of dietetics students and registered dieticians toward obesity. Journal of the American Dietetic Association, v.95, n.8, p.914-916 1995.

PAGAN, Jose A.; DAVILA, Alberto. Obesity, occupational attainment, and earnings. Social Science Quarterly, v.78, n.3, p. 756-770, 1997.

PEART, Naida D.; MARSH, Herbert W.; RICHARDS, Garry E. The Physical Self Description Questionnaire: furthering research linking physical self-concept, physical activity and physical education. In: AUSTRALIAN ASSOCIATION FOR RESEARCH IN EDUCATION. 2006. Disponível em: <http://www.aare.edu.au/05pap/pea05307.pdf>. Acesso em:20 mar.2017.

PIERCE, Jeanne Walsh; WARDLE, Jane. Cause and effect beliefs and self-esteem of overweight children. Journal of Child Psychology and Psychiatry, v.38, n.6, p.645-650, 1997.

PUHL, Rebecca M.; LUEDICKE, Joerg. Weight-based victimization among adolescents in the school setting: Emotional reactions and coping behaviors. Journal of Youth and Adolescence, v.41, n.1, p. 27-40, 2012.

ROTHBLUM, Esther D.; MILLER, Carol T.; GARBUTT, Barbara. Stereotypes of obese female job applicants. The International Journal of Eating Disorders, v.7, n.2, p.277-283, 1988.

SCARPA, Stefano et al. Peer-victimization during physical education and enjoyment of physical activity. Perceptual \& Motor Skills, v.115, p.319-324, 2012.

SCARPA, Stefano et al. Un contributo alla validazione italiana del Physical Self-Description Questionnaire short. II Giornale Italiano di Psicologia dello Sport, v.8, p.25-31, 2010. 
SCHWARTZ, Marlene B. et al. Weight bias among health professionals specializing in obesity. Obesity Research, v.11, n.9, p.1033-1039, 2003.

SIDANIUS, Jim; PRATTO, Felicia. Social dominance: An intergroup theory of social hierarchy and oppression. Cambridge : Cambridge University Press, 1999.

TEACHMAN, Bethany A., BROWNELL, Kelly D. Implicit anti-fat bias among health professionals: is anyone immune? International Journal of Obesity, v. 25, n. 10, p. 1525, 2001.

TEACHMAN, Bethany A. et al. Demonstration of implicit anti-fat bias: The impact of providing casual information and evoking empathy. Health Psychology, v. 22, n. 1, p. 68, 2003.

US DEPARTMENT OF HEALTH AND HUMAN SERVICES. The Surgeon General's Vision for a Healthy and Fit Nation. Rockville, 2010.

VARGAS, Patrick T.; SEKAQUAPTEWA, Denise; VON HIPPEL, William. Armed only with paper and pencil, "low-tech measures of implicit attitudes. In: B Wittenbrink, N Schwarz, Implicit measures of attitudes. New York: Guilford, 2007. p.103-119.

WHO. Obesity and overweight fact sheet, 2016. Disponível em: <http://www.who.int/ mediacentre/factsheets/fs311/en/>. Acesso em: 20 mar.2017.

WIGTON, Robert S.; MCGAGHIE, William C. The effect of obesity on medical students' approach to patients with abdominal pain. Journal of General Internal Medicine, v.16, n.4, p.262-265, 2001.

ZOGMAISTER, Cristina; CASTELLI, Luigi. La misurazione di costrutti impliciti attraverso l'Implicit Association Test. Psicologia sociale, v.1, p.65-94, 2006. 